\title{
Anomalous Wave Reflection at the Interface of Two Strongly Nonlinear Granular Media
}

\author{
V.F. Nesterenko, ${ }^{1,2, *}$ C. Daraio, ${ }^{2}$ E. B. Herbold, ${ }^{1}$ and S. Jin ${ }^{1,2}$ \\ ${ }^{1}$ Department of Mechanical and Aerospace Engineering, University of California at San Diego, La Jolla, California 92093-0411, USA \\ ${ }^{2}$ Materials Science and Engineering Program, University of California at San Diego, La Jolla, California 92093-0418, USA
}

(Received 15 June 2005; published 6 October 2005)

\begin{abstract}
Granular materials exhibit a strongly nonlinear behavior affecting the propagation of energy and information. Dynamically self-organized strongly nonlinear solitary waves are the main information carriers in granular chains. We report the first experimental observation of the dramatic change of solitary wave reflectivity from the interface of two granular media triggered by a magnetically induced precompression. It may be appropriate to name this phenomenon the "acoustic diode" effect. We explain this effect by the high gradient of particle velocity near the interface.
\end{abstract}

DOI: 10.1103/PhysRevLett.95.158702

Strongly nonlinear granular chains are "sonic vacuum" (SV) type systems that support a new type of solitary wave with parameters determined by the interaction force [1-3]. These solitary waves are qualitatively different from the well known weakly nonlinear solitary waves of the Korteweg-de Vries equation [4,5] which were first discovered experimentally by Russel [6] in 1834 . The concept of a SV was proposed to emphasize the uniqueness of the types of materials that do not support sound waves without initial prestress $[3,7]$. The unique property of these materials is that a single parameter (initial prestress) is able to tune the response from a linear to a strongly nonlinear regime. One of the main features of strongly nonlinear solitary waves is that the speed is strongly influenced by interrelated potential and kinetic energies [3]. A granular chain with particles interacting according to Hertz law [8] is just one example of strongly nonlinear behavior which can support the new type of solitary wave [3]. Different groups investigated numerically and experimentally the properties of these waves [1,2,9-20] and found a good agreement with the theoretical predictions based upon a long wave approximation. Nonlinear dynamic properties can be extended to other metamaterials including the propagation of electrical or other types of signals. Interesting applications of this new area of wave dynamics have been proposed, e.g., for the creation of nanodroplets [21].

Another intriguing property of these materials is the reflection of the solitary waves at the interface of two SV materials $[3,7,20,22]$, from imperfections [11] or from a wall [19]. Based on the former, the novel concept of impulse trapping inside a protecting granular laminar layer has been proposed [23,24].

In this Letter we present the first experimental and numerical observation of strongly nonlinear wave interaction with the interface of two SV-type systems resulting in anomalous reflected compression and transmitted rarefaction waves when the magnetically induced prestress is applied.

We propose a new method of noncontact precompression based on the magnetic interaction of the first magnetic particle in the chain with a Nd-Fe-B ring magnet [25]. The
PACS numbers: 05.65.+b, 43.25.+y, 45.70.-n, 46.40.Cd

magnetic force $(2.38 \mathrm{~N})$ is practically independent of the motion of the magnetic particle.

In experiments we placed a chain of 20 nonmagnetic stainless steel (316 type) particles (plus a magnetic particle on the top) with diameter $a=4.76 \mathrm{~mm}$ and mass $0.4501 \mathrm{~g}$ above 21 polytetrafluoroethylene (PTFE) beads with diameter $a=4.76 \mathrm{~mm}$ and mass $0.1226 \mathrm{~g}$. The elastic moduli and Poisson's ratios for PTFE and stainless steel were equal to $1.46 \mathrm{GPa}$ [26] and 0.46 and $193 \mathrm{GPa}$ and 0.3 , respectively. Piezogauges measuring the compression force [26] were placed in the 8th and 4th particle above the interface and in the 4th and 8th particle below the interface.

The equations of motion used in numerical calculations are similar to those in $[1,3]$. The intrinsic MATLAB ODE45 solver was used with a time step of $0.8 \mu \mathrm{s}$. No restitutional losses were taken into account. The linear momentum and energy were conserved with a relative error of $10^{-12} \%$ and $10^{-8} \%$. The curves obtained in numerical calculations represent the averaged compression forces on the contacts of each particle [26], except curves in Figs. 3(a) and 3(c) where the contact forces between particles are shown. The displacement $(\delta)$ of each particle is calculated from the equilibrium positions with no external load.

The results without magnetic precompression are presented in Fig. 1. A solitary wave ("I") was excited in the system by an impact of an alumina cylinder with a mass of $0.47 \mathrm{~g}$ and velocity of $0.44 \mathrm{~m} / \mathrm{s}$. This single solitary wave generates multiple pulses (" $T$ ") in the PTFE chain after the interaction with the interface. No reflected compression wave is detected in experiments or numerical calculations [Figs. 1(a) and 1(b)] as in [3,7,22-24].

The mechanism explaining the practically complete energy transfer into the PTFE chain and the absence of a reflected compression wave is illustrated by the numerical results in Fig. 1(c). The slopes of the displacement-time curves represent the velocity of the corresponding particles. It is evident that a series of gaps [Fig. 1(d)] open between the stainless steel particles in the vicinity of the interface. Therefore a "fracture wave" is propagating into the stainless steel chain similar to the one originating when the solitary wave arrives at the free surface [12] due to high 


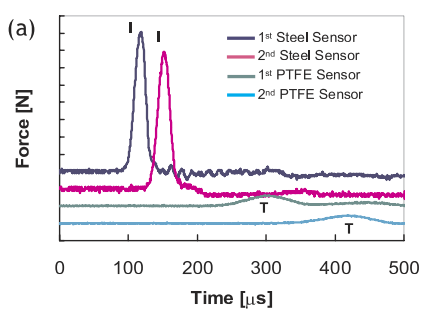

(c)

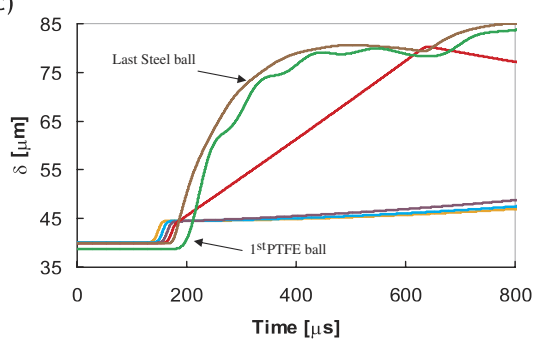

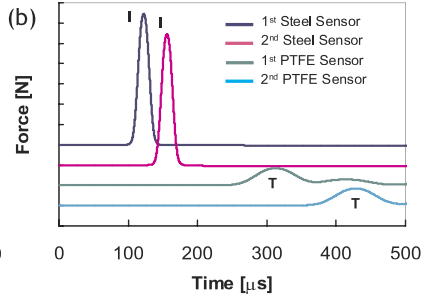

(d)

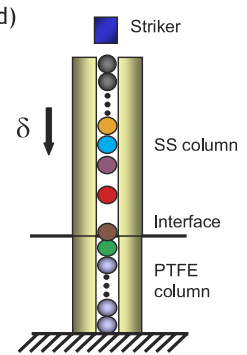

FIG. 1 (color online). Incident pulse $(I)$ interaction with the interface of the two SVs composed of 20 stainless steel and 21 PTFE beads. (a) Experimental data. (b) Numerical simulation of (a), vertical scales $1 \mathrm{~N}$ (a) and $2 \mathrm{~N}$ (b) per division. (c) Displacements $(\delta)$ of beads adjacent to the interface. (d) The relative positions of particles $600 \mu \mathrm{s}$ after the impact.

gradients of particle velocities. Gap openings are also observed in a 1D granular chain in the process of solitary wave collision, which is related to the generation of secondary solitary waves $[14,17,18]$, and in 2D numerical simulations of the transmission of the static force [27]. Gaps are also related to the subharmonics and noise excitation in the transmission of the acoustic wave in granular media [28].

The gap's opening and closing introduce an entirely new time scale in the system which is determined by the size of the gaps and particle velocities instead of the time of flight determined by particle diameter or length of the chain and signal speed.

The strongly nonlinear particle's interaction results in a high gradient of particle velocity in the incident wave that translates into a high gradient of velocity near the interface with the last stainless steel particle absorbing the main part of the energy. The zero tensile strength of the chain ensures the unidirectional energy transfer to the PTFE chain without sending any tensile wave back to the stainless steel chain. Thus, the observed behavior is due to a double nonlinearity: a strongly nonlinear compression part of the interacting force and a zero tensile strength of the system.

At the moment when the process of energy and linear momentum transmission into the PTFE chain is practically complete, only a very small portion of the impactor's kinetic energy (about $0.16 \%$ ) is reflected and the second steel particle is rebounding from the interface with a velocity of $0.018 \mathrm{~m} / \mathrm{s}$. Without the gravitational precompression this particle rebounds much later with a velocity significantly smaller $(0.0009 \mathrm{~m} / \mathrm{s})$ than in the previous case. This indicates that the reflected energy can be increased with the initial precompression.

The sequence of pulses in the PTFE chain is generated by the decelerating interfacial stainless steel particle, evident from the kinks of decreasing amplitude on the displacement curve of the first PTFE particle in Fig. 1(c).

The application of the magnetically induced precompression resulted in a completely different reflection of the strongly nonlinear pulse from the interface [compare Fig. 1(a) with Fig. 2(a) and Fig. 1(b) with Fig. 2(b)]. In this case the compression pulse is propagating into the steel chain followed by an oscillatory rarefaction wave [Figs. 2(a) and 2(b)]. The acoustic impedance of the stainless steel chain is about 1 order of magnitude higher than that of the PTFE chain. In the linear approach the incident compression solitary wave should result in a rarefaction wave propagating back from the interface into the stainless steel chain, it is indeed noticeable from the coordinated change of sign of the slope in the displacement curves starting from the 4th stainless steel particle from the interface [Fig. 2(c)]. Additional to the expected reflected rarefaction wave we observed experimentally and numerically anomalous reflected compression waves $(R)$ [Figs. 2(a) and 2(b)].

Based on the measurements of compression force inside particles [Fig. 2(a)] the leading reflected pulse in experiments has an amplitude (total contact force $F_{m}$ including initial preload $F_{0}$ ) of about one half of the amplitude $F_{m}$ of the incident wave. The relation between measured compression force inside particles and maximum contact force $F_{m}$ is discussed in [26]. It should be noted that the con-

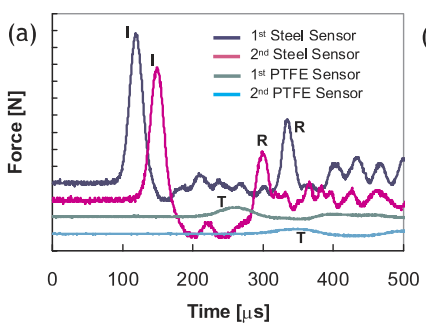

(c)

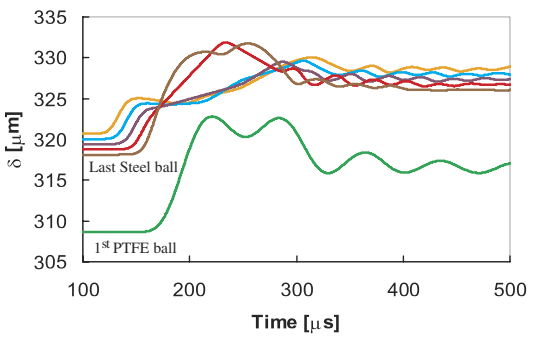

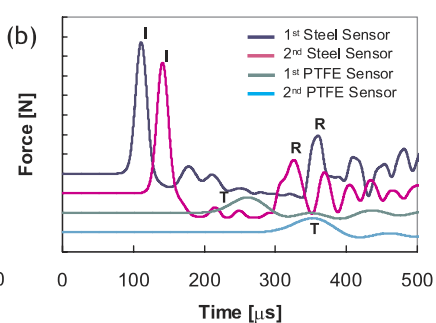

(d)

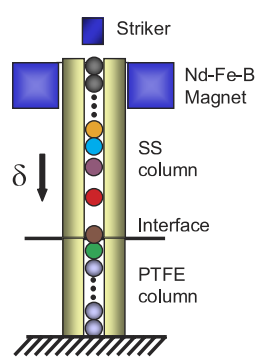

FIG. 2 (color online). Anomalous pulse reflection from magnetically preloaded interface of two sonic vacua in a configuration similar to Fig. 1. (a) Experimental data. (b) Numerical simulation of (a); vertical scales $1 \mathrm{~N}$ (a) and $2 \mathrm{~N}$ (b) per division. (c) Displacements $(\delta)$ of the stainless steel and PTFE beads adjacent to the interface related to the beginning of the formation of the rarefaction wave and anomalous reflected compression waves. (d) Relative positions of particles about $200 \mu$ s after the impact. 
tribution of the incident rarefaction wave to the reflected compression wave, based on the acoustic impedance ratio, cannot bring the force above the initial precompression. Also, the leading transmitted $(T)$ compression pulse in the PTFE chain is followed by unexpected rarefaction pulses [Figs. 2(a) and 2(b)].

We may say that a superimposed static precompression on the interface of two sonic vacua made this interface visible based on the reflected signal in contrast to the uncompressed case. A peculiar characteristic of the leading reflected compression wave is related to its delayed time of arrival (about $90 \mu \mathrm{s}$ ) at the gauges in experiments and calculations based on the distance from the interface and the speed of the incident and reflected compression pulses [Figs. 2(a) and 2(b)].

When the leading reflected compression wave is formed, at $500 \mu \mathrm{s}$ after the impact, the energy transferred to the PTFE chain is about $86 \%$ of the combined kinetic energy of the striker and the energy supplied by the magnetic force at the early stage of the motion of the first particle (about $25 \mu \mathrm{s}$ ). This contrasts with the previous case where almost all of the kinetic energy of the striker (over 99\%) was transferred into the PTFE chain. It may be appropriate to name this dramatic change of reflectivity triggered by the initial precompression (zero and 14\% reflected energy, respectively) the "acoustic diode" effect.

The reflected compression waves were formed mainly by the rebounding motion of the last stainless steel particle due to the resistance of the PTFE beads. This phenomenon is a clear manifestation of the discrete nature of the system and cannot be captured in a continuum approximation similar to the fracture of a chain in the vicinity of impacted end $[1,3]$ or the formation of secondary solitary waves $[14,17,18]$. The particles in the vicinity of the interface are self organizing into a state close to the original precompressed state [Fig. 2(c)].
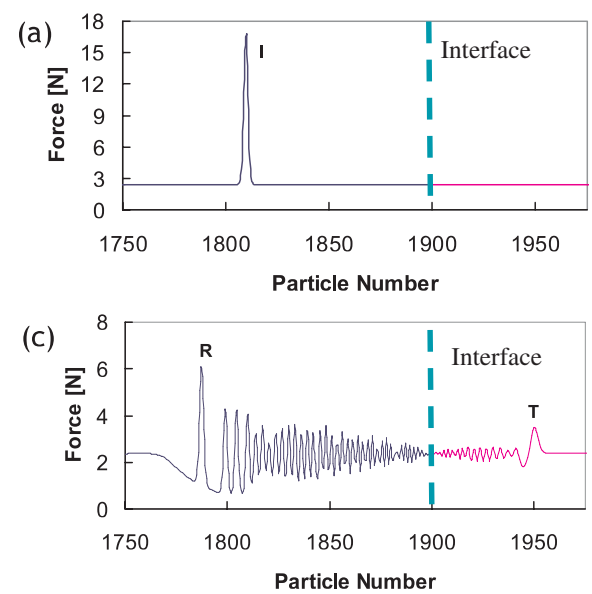

As in the previous case [Fig. 1(c)], the stainless steel interfacial particle serves as the main energy transformer from the stainless steel chain to the PTFE chain [Fig. 2(c)]. Again, a fracture wave follows the rarefaction wave that is propagating from the interface back into the stainless steel chain. In the case of stronger precompression the gaps are closed rather quickly assisting the formation of reflected compression pulses. The opening and closing of gaps introduce a new time scale which is much shorter than in the previous case due to the significantly smaller size of the opened gaps and larger velocities of the particles [compare Figs. 1(c) and 2(c)].

Initial precompression in a counterintuitive manner triggers the generation of a reflected compression wave and does not suppress the process of gaps opening and closing, but instead makes it faster. Their characteristic opening and closing also participate in the process of wave reflection in this case [Figs. 2(c) and 2(d)]. This indicates that the response of the interface between two SVs can be qualitatively tuned by the applied static preloading.

The gap openings between the stainless steel particles in the vicinity of the interface in the investigated setup has a threshold on the amplitude of the incident wave detected in numerical calculations. This threshold amplitude of contact force $F_{m}$ is approximately equal to $4.3 \mathrm{~N}$, which is larger than the static preload at the interface $2.473 \mathrm{~N}$. The anomalous reflected compression wave was generated at this amplitude even when no gap openings were detected. This shows that the anomalous reflected compression wave is due to the changing direction of the velocity of the last stainless steel particle.

To identify the possible influence of the incident rarefaction wave on the reflected compression pulse in experiments, the interaction of a single solitary wave $(I)$ with similarly preloaded $(2.38 \mathrm{~N})$ sonic vacua was simulated. It was formed in a stainless steel chain with a larger number
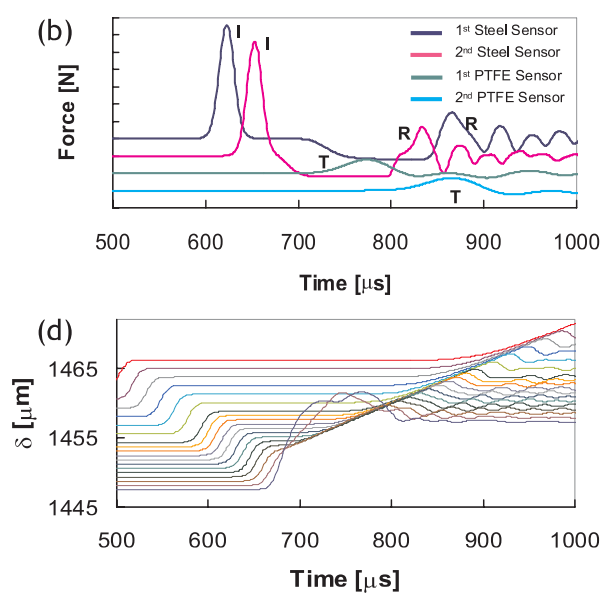

FIG. 3 (color online). (a) Incident solitary wave $(I)$. (b) The forces inside the stainless steel particles corresponding to the positions of the gauges in experiments close to the interface, vertical scale is $2 \mathrm{~N}$ per division [compare Fig. 3(b) with Figs. 2(a) and 2(b)]. (c) The reflected rarefaction wave, anomalous compression solitary wave $(R)$, and trailing pulses in the stainless steel chain and transmitted compression pulse $(T)$ with the rarefaction pulse and oscillatory tail in the PTFE chain. (d) Displacements of the stainless steel beads adjacent to the interface. 
of particles (1900) [Fig. 3(a)] after the impact by an alumina striker with a mass equal to the mass of the stainless steel particle and a velocity of $0.44 \mathrm{~m} / \mathrm{s}$. The incident rarefaction wave followed far behind the solitary wave and did not participate in the considered soliton interaction with the interface.

After the interaction, an expected reflected rarefaction wave formed close to the interface in the stainless steel chain and was followed by a compression pulse and an oscillatory tail [Figs. 3(b) and 3(c)]. The amplitude of the first reflected compression pulse [Fig. 3(b)] and the behavior of the displacements of the particles adjacent to the interface [Fig. 3(d)] were very similar to our experiments and numerical calculations for a smaller number of particles where the incident rarefaction wave was present [Figs. 2(b) and 2(c)]. This clearly indicates that the observed anomalous reflected compression pulse and trailing oscillatory tail are caused by the incident solitary wave.

The reflected compression solitary wave is a nonlinear wave with a speed apparently higher than the speed of the head of the reflected rarefaction wave and oscillating tail. It becomes the leading reflected pulse about 1000 particles from the interface as was found in separate numerical calculations with 25000 stainless steel and 1000 PTFE particles. It is interesting that only one anomalous reflected compression solitary pulse was able to overcome the head of the reflected rarefaction wave, with amplitude $F_{m}$ decreasing by about $40 \%$ during this process. As a result, the reflected signal at a relatively large distance from the interface (larger than 1000 particles) is composed of three essential elements in the following order: leading anomalous reflected compression solitary wave, a rarefaction wave, and an oscillatory tail. On later stages the oscillatory tail overcomes the head of the rarefaction wave leaving only a leading reflected compression solitary wave and an oscillatory tail. The leading transmitted compression pulse in the PTFE chain is followed by an unexpected rarefaction wave and an oscillatory tail [Figs. 3(c) and 3(d)], also evolving into a leading transmitted compression solitary wave and an oscillatory tail.

We did not observe a qualitative change of the reflectivity under the applied precompression when the wave approached the interface from the PTFE side.

In summary we observed a strong sensitivity to the initial precompression of the reflected and transmitted energy from the interface of the two granular media. This phenomenon can be named the acoustic diode effect. It can be employed for designing tunable information transportation lines with the unique possibility to manipulate the signals delay and reflection at will, and decompositions/ scrambling of security-related information. It can also be used for identification of such interfaces (e.g., geological multilayer structures consisting of dissimilar granular materials) and for optimization of shock protection layers composed of uniquely combined composite granular media with layers of different particle sizes (masses) and elastic constants.
The authors wish to acknowledge the support of this work by the U.S. NSF (Grant No. DCMS03013220).

*Electronic address: vnesterenko@ucsd.edu

[1] V.F. Nesterenko, Prikl. Mekh. Tekh. Fiz. 24, 136 (1983) [J. Appl. Mech. Tech. Phys. 24, 733 (1984)].

[2] A. N. Lazaridi and V.F. Nesterenko, Prikl. Mekh. Tekh. Fiz. 26, 115 (1985); [J. Appl. Mech. Tech. Phys. 26, 405 (1985)].

[3] V.F. Nesterenko, Dynamics of Heterogeneous Materials (Springer-Verlag, New York, 2001), Chap. 1.

[4] D. J. Korteweg and G. de Vries, London, Edinburgh and Dublin Philosophical Magazine and Journal of Science 39, 422 (1895).

[5] M. Remoissenet, Waves Called Solitons (Concepts and Experiments) (Springer-Verlag, Berlin, 1999), 3rd ed.

[6] J.S. Russel, Report on Waves, Report of the 14th Meeting of the British Association for the Advancement of Science, York, 1844, p. 311.

[7] V. F. Nesterenko, J. Phys. IV 4, C8-729 (1994).

[8] H. Hertz, J. Reine Angew. Math. 92, 156 (1881).

[9] C. Coste, E. Falcon, and S. Fauve, Phys. Rev. E 56, 6104 (1997).

[10] M. Manciu, S. Sen, and A.J. Hurd, Physica A (Amsterdam) 274, 588 (1999).

[11] M. Manciu, S. Sen, and A. J. Hurd, Physica A (Amsterdam) 274, 607 (1999).

[12] E. J. Hinch and S. Saint-Jean, Proc. R. Soc. A 455, 3201 (1999).

[13] C. Coste and B. Gilles, Eur. Phys. J. B 7, 155 (1999).

[14] M. Manciu, S. Sen, and A.J. Hurd, Phys. Rev. E 63, 016614 (2001).

[15] E. Hascoet and H. J. Herrmann, Eur. Phys. J. B 14, 183 (2000).

[16] S. Sen and M. Manciu, Phys. Rev. E 64, 056605 (2001).

[17] F. S. Manciu and S. Sen, Phys. Rev. E 66, 016616 (2002).

[18] S. Sen, S. Chakravarti, D. P. Visco, Jr., D. T. Wu, M. Nakagawa, and J.H. Agui, Jr., in Modern Challenges in Statistical Mechanics, AIP Conf. Proc. No. 658, edited by V. M. Kenkre and K. Lindenberg (AIP, Melville, NY, 2003), p. 357.

[19] S. Job, F. Melo, S. Sen, and A. Sokolow, Phys. Rev. Lett. 94, 178002 (2005).

[20] L. Vergara, Phys. Rev. Lett. 95, 108002 (2005).

[21] S. Sen, M. Manciu, and F. S. Manciu, Appl. Phys. Lett. 75, 1479 (1999).

[22] V.F. Nesterenko, A. N. Lazaridi, and E. B. Sibiryakov, Prikl. Mekh. Tekh. Fiz. 36, 19 (1995) [J. Appl. Mech. Tech. Phys. 36, 166 (1995)].

[23] J. Hong and A. Xu, Appl. Phys. Lett. 81, 4868 (2002).

[24] J. Hong, Phys. Rev. Lett. 94, 108001 (2005).

[25] C. Daraio, V.F. Nesterenko, E. B. Herbold, and S. Jin, http://arxiv.org/abs/cond-mat/0506513.

[26] C. Daraio, V.F. Nesterenko, E. B. Herbold, and S. Jin, Phys. Rev. E 72, 016603 (2005).

[27] C. Goldenberg and I. Goldhirsch, Nature (London) 435 , 188 (2005).

[28] V. Tournat, V. E. Gusev, and B. Castagnede, Phys. Lett. A 326, 340 (2004). 